\title{
METODOLOGÍA PARA LA IMPLEMENTACIÓN DE UN OPERADOR INTELIGENTE SOBRE TARJETA DE DESARROLLO QUE PERMITA LA CLASIFICACIÓN DE INDIVIDUOS SANOS Y ENFERMOS CON FENOTIPOS METABÓLICOS SOBREPESO Y OBESOS.
}

\section{METHODOLOGY FOR THE IMPLEMENTATION OF AN INTELLIGENT OPERATOR ON DEVELOPMENT CARD THAT ALLOWS THE CLASSIFICATION OF HEALTHY AND SICK INDIVIDUALS WITH METABOLIC PHENOTYPES OVERWEIGHT AND OBESES.}

\author{
PhD. Gerardo Chacón", MSc. Oscar J. Suarez ${ }^{* *}$, Duran, Leyder ${ }^{* * *}$, Joseph \\ Sequeda****, Juan Tarazona***
}

${ }^{*}$ Grupo de Investigación Automatización y Control, Pamplona, Colombia. Correo: jose.chacon@unipamplona.edu.co.

** Centro de Investigación y de Estudios Avanzados del IPN Av. del Bosque 1145, Col. El Bajío, Zapopan, Jalisco, 45019, Mexico.

**** Universidad Simón Bolívar, Facultad de Ingeniería, Cúcuta 540004, Colombia. Correo: j.chacon@unisimonbolivar.edu.co. ${ }^{* * * *}$ Semillero investigación Ciencias Computacionales, , Pamplona, Colombia. Correo:_sequeda_94@hotmail.com

Resumen: La obesidad y el sobrepeso son unas patología que ha estado aumentando a un ritmo acelerado en las últimas décadas y ahora ha alcanzado proporciones epidémicas. Datos recientes sugieren que el $65 \%$ de los adultos tienen sobrepeso (índice de masa corporal $>25 \mathrm{~kg} \mathrm{~m} \mathrm{-2}$ ) y el $30 \%$ son obesos (índice de masa corporal > $30 \mathrm{~kg} \mathrm{~m}-2$ ). Estas patologías se asocia con numerosas complicaciones metabólicas como Diabetes tipo 2, Dislipidemia, Hipertensión, enfermedades cardiovasculares y varias formas de cáncer. Se postula actualmente que no todos los individuos que muestran fenotipos metabólicos de obesos y con sobrepeso tienen que considerarse como individuos patológicos. Un alto porcentaje de los individuos estudiados que presentan estos fenotipos no muestran las complicaciones metabólicas habituales. El objetivo general de esta investigación es desarrollar una metodología para la implementación de un operador inteligente en tarjeta de desarrollo que permita hacer una clasificación de individuos sanos y enfermos considerando los fenotipos metabólicos sobrepeso y obesos. El resultado de esta investigación es un protocolo de desarrollo de una aplicación que permita diagnosticar con un porcentaje aceptable de error los individuos sanos y enfermos en los fenotipos mencionados y además sirva como apoyo a los especialistas de la salud en el diagnóstico de este tipo de patologías.

Palabras clave: Hipertensión, Operadores Inteligentes , Fenotipos Metabólicos..

\begin{abstract}
Obesity and overweight are a pathology that has improved and accelerated in the latest trends and has now reached epidemic proportions. The body mass index (body mass index $>25 \mathrm{~kg} \mathrm{~m} \mathrm{-2)} \mathrm{and} \mathrm{30 \%} \mathrm{are} \mathrm{obese} \mathrm{(body} \mathrm{mass} \mathrm{index}>30 \mathrm{~kg} \mathrm{~m} \mathrm{-2).} \mathrm{These}$ pathologies are associated with numerous metabolic complications such as type 2 diabetes, dyslipidemia, hypertension, cardiovascular diseases and various forms of cancer. It is currently published that not all individuals who show metabolic phenotypes of obese and overweight are considered as pathological individuals. A high percentage of the
\end{abstract}


individuals studied who present these phenotypes do not show the usual metabolic complications. The general objective of this research is to develop a methodology to develop an intelligent communication. The result of this research is a protocol for the development of an application that can be diagnosed with an acceptable percentage of error by healthy and sick individuals in the phenotypes in which they are used and in which health specialists can be helped. the diagnosis of this type of pathologies.

Keywords: Hypertension, Intelligent Operators, Metabolic Phenotypes

\section{INTRODUCCIÓN}

Las Redes Neuronales Artificiales, NNET (Artificial Neural Networks) están inspiradas en las redes neuronales biológicas del cerebro humano. Están constituidas por elementos que se comportan de forma similar a la neurona biológica en sus funciones más comunes. Estos elementos están organizados de una forma parecida a la que presenta el cerebro humano. (Olabe, 1998). Las ANN al margen de "parecerse" al cerebro presentan una serie de características propias del cerebro. Por ejemplo, las NNET aprenden de la experiencia, generalizan de ejemplos previos a ejemplos nuevos y abstraen las características principales de una serie de datos. Aprender: adquirir el conocimiento de una cosa por medio del estudio, ejercicio o experiencia. Las NNET pueden cambiar su comportamiento en función del entorno. Se les muestra un conjunto de entradas y ellas mismas se ajustan para producir unas salidas consistentes. Figura 1 NNET

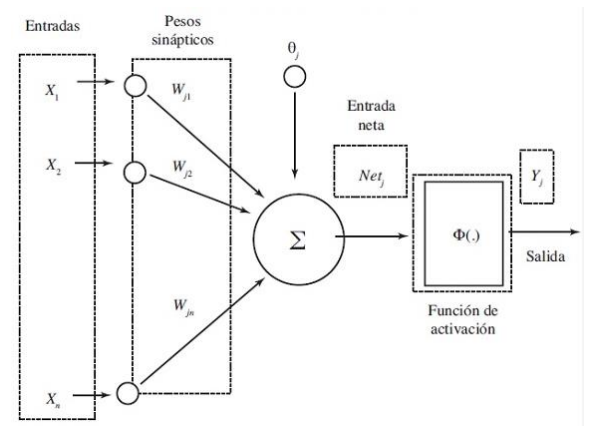

Figura 1Modelo NNET

Fuente Caicedo y López 2009

Las NNET son redes interconectadas masivamente en paralelo de elementos simples (usualmente adaptativos) y con organización jerárquica, las cuales intentan interactuar con los objetos del mundo real del mismo modo que lo hace el sistema nervioso biológico, (Olabe, 1998). Debido a su fundamentación biológica, las redes neuronales artificiales presentan un gran número de características semejantes a las del cerebro, por ejemplo, son capaces de aprender de la experiencia, de generalizar de casos anteriores a nuevos casos, de abstraer características esenciales a partir de entradas que representan información irrelevante, entre otros ejemplos. Debido a esto, las redes neuronales artificiales ofrecen numerosas ventajas y son aplicadas en múltiples áreas. Dentro de las ventajas que las redes neuronales pueden ofrecer están: Aprendizaje Adaptativo. Capacidad de aprender a realizar tareas basadas en un entrenamiento $o$ en una experiencia inicial. Autoorganización. Una red neuronal puede crear su propia organización o representación de la información que recibe mediante una etapa de aprendizaje. Tolerancia a fallos. La destrucción parcial de una red conduce a una degradación de su estructura; sin embargo, algunas capacidades de la red se pueden retener, incluso sufriendo un gran daño. Operación en tiempo real. Los cómputos neuronales pueden ser realizados en paralelo; para esto se diseñan y fabrican máquinas con hardware especial para obtener esta capacidad. Fácil inserción dentro de la tecnología existente. Se pueden obtener chips especializados para redes neuronales que mejoran su capacidad en ciertas tareas. Ello facilitará la integración modular en los sistemas existentes. La distribución de neuronas dentro de la red se realiza formando niveles o capas, con un número determinado de dichas neuronas en cada una de ellas. A partir de su situación dentro de la red, se pueden distinguir tres tipos de capas:

En 1986, Rumelhart, Hinton y Williams, formalizaron un método para que una red neuronal aprendiera la asociación que existe entre los patrones de entrada y las clases correspondientes, utilizando varios niveles de neuronas (Hilera J, 2000).

Support Vector Machines o Máquinas de Soporte Vectorial (SVM) es una poderosa metodología para resolver problemas de clasificación no lineales, estimación de funciones y estimación de densidades que también ha llevado a muchos otros desarrollos recientes en los métodos de aprendizaje basados en kernel en general. A diferencia de las Redes Neuronales Artificiales, que utilizan el 
principio de Minimización del Riesgo Empírico (ERM) durante la fase de entrenamiento, las SVMs se basan en el principio de Minimización del Riesgo Estructural (SRM), la cual ha mostrado un mejor desempeño que el ERM, puesto que minimizan un límite superior al riesgo esperado a diferencia del ERM que minimiza el error sobre los datos de entrenamiento. (Colmenares, 2009). Figura 2 modelo SVM

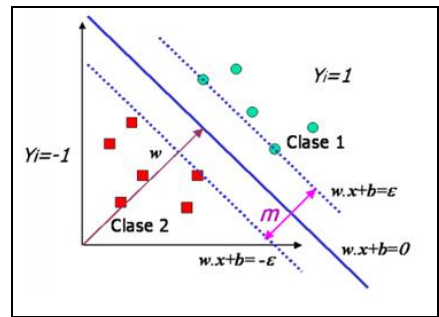

Figura 2 Modelo SVM

Fuente Colmenares 2009

De acuerdo con la OMS (Organización Mundial de la Salud), la obesidad es una enfermedad crónica caracterizada por el aumento de la grasa corporal, que se asocia a un mayor riesgo para la salud. (Moreno, 2012).

La clasificación actual de la obesidad propuesta por la OMS, (Moreno, 2012). está basada en el Índice de Masa Corporal (IMC), que corresponde a la relación entre el peso expresado en kilos y el cuadrado de la altura, expresada en metros $(\mathrm{kg} / \mathrm{m} 2)$. El efecto de la obesidad sobre la enfermedad coronaria depende en cierto modo de otras complicaciones que derivan de la obesidad, como resistencia a la insulina, la diabetes, la dislipidemia o la hipertensión arterial. Sin embargo, la obesidad ejerce un efecto en la mortalidad por cardiopatía isquémica incluso tras el ajuste por otros factores de riesgo cardiovascular. En un meta análisis de 57 estudios prospectivos sobre la influencia del IMC en la mortalidad, se objetivó que por cada incremento de $5 \mathrm{~kg} / \mathrm{m} 2$ del IMC por encima de $25 \mathrm{~kg} / \mathrm{m} 2$ el riesgo de muerte por enfermedad aumentaba un $40 \%$. Otra de las consecuencias de la obesidad es la insuficiencia cardiaca. El análisis de 5.881 sujetos del Framingham Heart Study demostró que el riesgo de insuficiencia cardiaca aumenta un $5 \%$ en los varones y un $7 \%$ en las mujeres por cada incremento de $1 \mathrm{~kg} / \mathrm{m} 2$ del IMC, tras el ajuste por otros factores de riesgo. El objetivo general de esta investigación es desarrollar una metodología para la implementación de un operador inteligente (OI) en tarjeta de desarrollo (TD) que permita hacer una clasificación de individuos sanos y enfermos considerando los fenotipos metabólicos sobrepeso y obesos El desarrollo está conformado en tres fases. En la primera se realiza la normalización de la base de datos y selección del OI inteligente y la TD utilizando el lenguaje Python. La segunda fase se desarrolla el OI, se prueba y valida en aplicación de escritorio. Finalmente se implementa el OI en tarjeta de desarrollo caso práctico: Clasificación de individuos sanos $\mathrm{y}$ enfermos con fenotipos metabólicos sobrepeso y obesos.

\section{Metodología}

La metodología propuesta se muestra en la figura 3

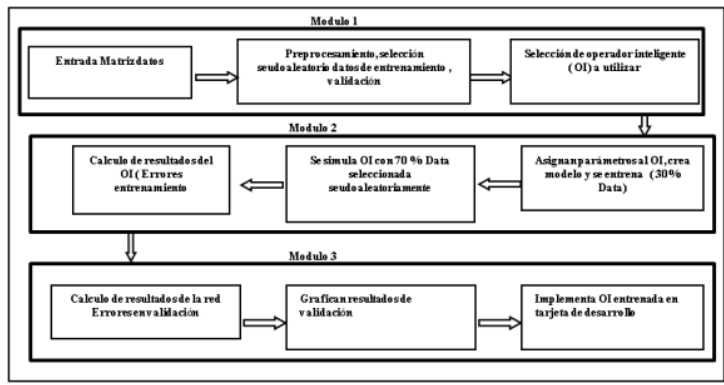

Figura 3 Metodología de desarrollo Fuente los autores

El Modulo 1 está formado por 3 etapas: La primera es la entrada de los datos que servirán en la investigación y se utilizaran en el desarrollo de la aplicación de escritorio para entrenar y validar. La segunda etapa es el preprocesamiento de la data donde se seleccionan variables a utilizar, se normaliza, se hace el escalamiento entre los valores de 0 y 1 y se seleccionan aleatoriamente los datos de entrenamiento y validación. La etapa 3 se selecciona el OI y la TD donde se implementara la aplicación. El modulo 2 consta igualmente de 3 etapas: La primera asigna parámetros al OI, se crea el modelo y se procede a realizar el entrenamiento con el $30 \%$ de la data. En la segunda etapa se simula el modelo de IO con el $70 \%$ de la data y en la etapa 3 se calculan errores en el entrenamiento finalmente, el modulo tres valida los resultados del OI a través de las métricas estadísticas, grafica resultados de las mismas y finalmente se implementa el OI en la tarjeta de desarrollo.

Para la implementación de esta metodología en esta investigación se utiliza la base de datos del estudio de prevalencia del síndrome metabólico de la ciudad de Maracaibo denominada PSMEM, esta es única en cuanto a que combina entrevistas y exámenes físicos y constituye un importante estudio del Centro De investigación de la 
Universidad del Zulia (LUZ) y tiene la responsabilidad de producir estadísticas vitales y de salud en el estado Zulia. Algunas de las enfermedades, condiciones médicas e indicadores de salud que se encuentran en PSMEM son Anemia, Enfermedades cardiovasculares, Diabetes y enfermedades de las extremidades inferiores, Exposiciones Ambientales, Nutrición y Obesidad. $\mathrm{Se}$ proponen dos modelos de $\mathrm{OI}$ : El primero una ANN con FFBP , la cual está formada por: una capa de entrada constituida por 53 neuronas, una por cada variable de entrada tomada de PSMEM. Una capa intermedia conformada por 8 neuronas asignadas a priori, (Ramírez C. 1997), (Haykin S 1999), (Cybenko G. 1989), activadas mediante funciones radiales $\varphi($.$) , de carácter no lineal con$ sus centros gravitacionales propios y finalmente una capa de salida con dos neurona cuyas función de activación son de límite duro simétrico $\varphi($. con respuesta 1 para obesos sanos y -1 para obesos enfermos . El algoritmo de entrenamiento utilizado es por lotes donde actualiza sólo los pesos y el umbral de la red después que el grupo de entrenamiento completo se ha aplicado a la red. Se suman los gradientes calculados en cada ejemplo de entrenamiento para determinar el cambio en los pesos y el umbral. El segundo Modelo es una SVM y para construirlo necesitamos dos parámetros adicionales: Gamma que es el parámetro de regularización, que determina el compromiso entre el ajuste del error de minimización y la suavidad, este se asigna como 10, y el sigma cuadrado que es el ancho de banda de la función gaussiana utilizada como kernel, este se asigna 0,2.

Para la selección de de la tarjeta de desarrollo se realizo una tabla comparativa entre las diferentes características de las tarjetas de desarrollo en el mercado, esta se muestra en la tabla 1.

Arduino es mejor para: proyectos de propósito simple. Por ejemplo, un sistema en el que el secador de ropas envía un mensaje de texto cuando estas están listas, o un sistema de video. Su uso es más práctico y mejor aprovechado para interactuar con objetos en el mundo real. Si la aplicación requiere conexión a Internet, tener un display multi-touch y completa automatización, el Arduino probablemente (Aranda F. 2011).

Raspberry $\mathrm{Pi}$ es mejor para: proyectos que requieren interfaz gráfica o internet. Ya que sus orí-genes son educativos, es también mejor aprovechado para principiantes que buscan un proyecto de computación educativo de bajo costo. Debido a sus varias entradas y salidas, también tiende a ser una plataforma preferida para proyectos multimedia como el XBMC Media Center o un cen-tro de juego. (Riaño, A 2014).

Beaglebone es mejor para: proyectos que puedan necesitar más procesamiento y rapidez y para los cuales la plataforma Arduino puede ser limitada, pero también que no exijan el procesamiento grafico que solo la plataforma Raspberry pi puede ofrecer. Esta plataforma tiene puerto para conectar una amplia gama de sensores, es perfecto para proyectos avanzados con interfaz al mundo real. También para proyectos que se quieran comercializar, ya que el Raspberry pi tiende más hacia un ecosistema closed-source, es imposible hacer propias versiones del mismo, mientras que en esta plataforma se puede tomar las características más importantes y usarlas en un diseño propio.

Se tomo la Raspberry $\mathrm{Pi}$ ya que la revisión documental de la literatura especializada reporta que el uso de esta para proyectos que requieren interfaz gráfica o internet ya que sus orígenes son educativos, tiene ventajas en cuanto a sus varias entradas y salidas, también tiende a ser una plataforma preferida para proyectos multimedia como el XBMC Media Center o un centro de juego (Riaño, A 2014).

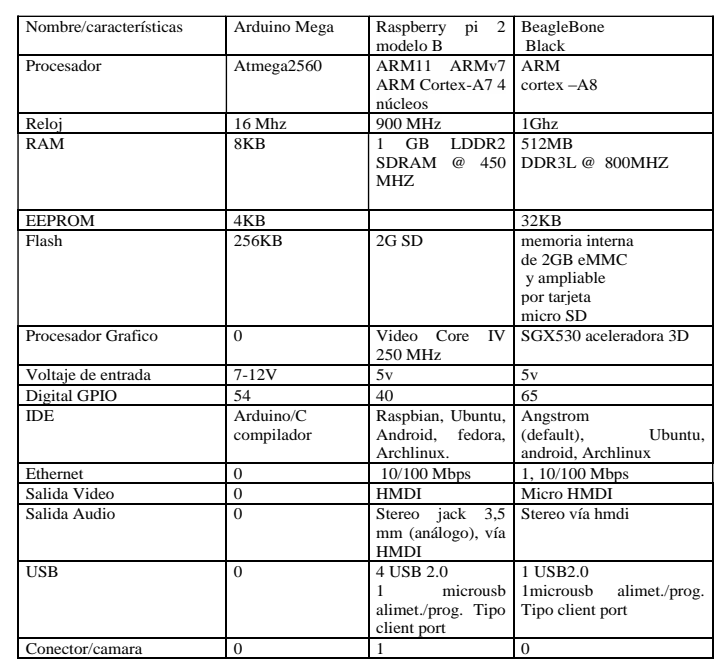

Tabla 1 Comparación de características de tarjetas de desarrollo. Fuente los autores.

Resultados.

Los resultados posibles se expresan en los términos representados en la tabla 2. Y los valores de los mismos en la tabla 3. Esto es: Primer caso, verdaderos positivos (VP), estos se consideran dos 
casos, los clasificados como sanos y en realidad están sanos (SS) y los clasificados como enfermos cuando en realidad están enfermos (EE). Segundo caso Falsos positivos representan los clasificados como enfermos cuando en realidad están sanos (ES) y sanos cuando en realidad están enfermos (SE). (Mandal 2015)

\begin{tabular}{|c|l|}
\hline VP & Verdaderos positivos \\
\hline FP & Falsos positivos \\
\hline SS & Clasificados como sanos y en realidad estan sanos \\
\hline EE & Clasificados como enfermos y en realidad estan enfermos \\
\hline ES & Clasificados como enfermos y en realidad estan sanos \\
\hline SE & Clasificados como sanos y en realidad estan enfermos \\
\hline
\end{tabular}

Tabla 2 posibles casos de clasificación. Fuente los autores.

\begin{tabular}{|c|c|c|c|}
\hline \multicolumn{3}{|c|}{ Clasificacion de los dos modelos } \\
\hline \multicolumn{2}{|c|}{ NNET } & \multicolumn{2}{c|}{ SVM } \\
\hline \multicolumn{2}{|c|}{ Primer caso } & \multicolumn{2}{c|}{ VP } \\
\hline \multicolumn{2}{|c|}{ VP } & SS & EE \\
\hline SS & EE & $91,20 \%$ & $90,10 \%$ \\
\hline $88,70 \%$ & $87 \%$ & \multicolumn{2}{c|}{ Segundo caso } \\
\hline \multicolumn{2}{|c|}{ Segundo caso } & \multicolumn{2}{c|}{ FP } \\
\hline \multicolumn{2}{|c|}{ FP } & ES & SE \\
\hline ES & SE & $8,80 \%$ & $9 \%$ \\
\hline $11,70 \%$ & $13 \%$ & & \\
\hline
\end{tabular}

Tabla 3 Resultados de la clasificación por los dos OI. Fuente los autores.

Se observa que los mejores resultados se obtienen con el OI SVM ya que los VP representan el $91,20 \%$ (sensibilidad) para los SS y 90,10\% ( especificidad) para los EE comparándolos con los respectivos $\mathrm{FP}$ son menores que para el caso del OI NNET.

Para la implementación de escritorio para ambos modelos se utiliza el software Matlab15, (Mathworks, 2015) específicamente para en el caso SVM se utilizar el Toolbox LS-SVM el cual es necesario seguir una serie de pasos,figura 4.

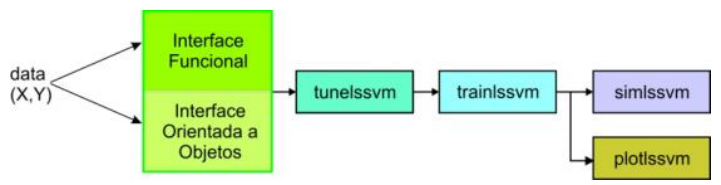

Fig 4. Secuencia de funciones del toolbox LSSVM. (Mathworks, 2008)

El primer paso es escoger el tipo de interface para la comunicación con las rutinas entre: funcional $\mathrm{u}$ orientada a objetos. El segundo paso es seleccionar los parámetros de ajuste adecuados (tunelssvm). El tercer paso es entrenar el modelo de LS-SVM empleando los parámetros anteriormente escogidos (trainlssvm). (Flores . 2002). El cuarto paso es Simular el modelo con la data de validación y visualizar mediante un gráfico los resultados, cuando sea posible (plotlssvm). El toolbox de LSSVM está destinado principalmente al uso con el paquete comercial Matlab. La caja de herramientas Matlab se compila y se prueba para diferentes arquitecturas de ordenadores incluyendo Linux y Windows. La mayoría de las funciones pueden manejar conjuntos de datos de hasta 20.000 puntos de datos o más.

A continuación se muestra del código fuente de la aplicación del clasificador en MatLab: La primera parte de la secuencia en MatLab corresponde a la carga de datos a partir de un archivo .txt contentivo de los datos de entrenamiento y los datos de prueba.

$>$ \% cargar dataset..

$>$ datos = load('Datos PSMEM .txt');

$>\mathrm{X}=$ datos $(1: 30,2: 6)$

$>\mathrm{Y}=\operatorname{datos}(1: 30,1)$

A continuación, la aplicación debe establecer los valores de los parámetros gamma, sigma2 y el tipo de tarea (clasificador/estimación de funciones) a ejecutar.

$$
\begin{aligned}
& >>\operatorname{gam}=10 ; \\
& >>\operatorname{sig} 2=0.2 ; \\
& >>\text { type }=\text { 'classification'; } \\
& \text { > } \quad \text { [alpha,b] } \\
& \text { trainlssvm }(\{X, Y, \text { type,gam,sig2,'RBF_kernel' }\})
\end{aligned}
$$

Luego de entrenar la SVM, se procede a su simulación para confrontar primero los datos de entrenamiento y luego los datos de prueba:

$>\mathrm{Xt}=$ datos $(31: 68,2: 6)$;

$>\mathrm{Yt}=$ datos $(31: 68,1)$;

> Ytest=simlssvm( $\{X, Y$, type,gam,sig2,'RBF_kern el','preprocess' $\},\{$ alpha,b $\}, X t)$;

En la Figura 5, se muestran los resultados de clasificar los patrones de entrenamiento, y se demuestra que los primeros casos fueron correctamente clasificados como enfermos (1) y los últimos casos como sano $(-1)$. 


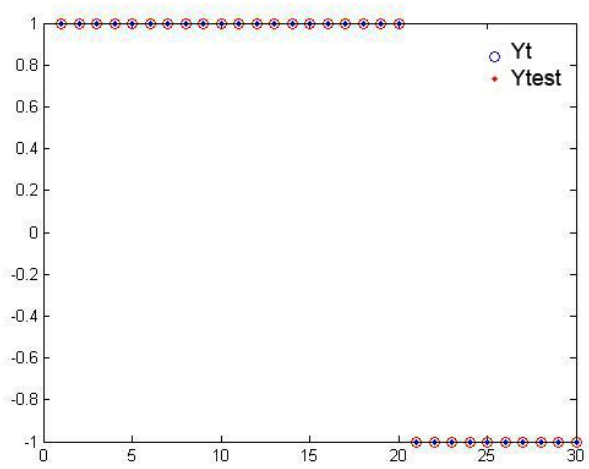

Fig. 5. Clasificación de la data de entrenamiento. Fuente los autores

\section{Antecedentes de algoritmos en aplicaciones biomédicas.}

Algunos investigadores se han dedicado al estudio de los algoritmos de inteligencia artificial tanto en el estudio biomédico como en la implementación de minicomputadores (Chang, \& Lin, 2011) Presentan el diseño y la implementación de una máquina de vector de soporte (SVM) sobre un dispositivo lógico programable (PLD). $\quad \mathrm{La}$ maquina es utilizada para solucionar el problema de la XOR la cual la implementaron sobre la tarjeta FPGA (ALTERA® FLEX10K) (Chongo 2016) [16]. Más adelante (Raponi, Bonnin (2011), plantearon la realización de un dispositivo de rehabilitación visual basado en sistemas embebidos del tipo ARM. Este proyecto lo realizaron utilizando la mini computadora Beaglebone Black, para adquirir y procesar en tiempo real señales de video provenientes de una mini-cámara portátil, y generar un patrón de estimulación visual que brinde un cierto grado de rehabilitación a personas con baja visión Carignano F 2011, e-Health Sensor Platform: Diseño e implementación de una plataforma sensorial biométrica para diagnóstico de pacientes en tiempo real y otras aplica-ciones médicas. (Martin, 2014) [13]. , cuyo proyecto tuvo un gran avance en la biomedicina, quienes realizaron un dispositivo de apoyo en el diagnostico medico el permite a los usuarios realizar un monitoreo del cuerpo utilizando las tarjetas Arduino y Beaglebone black y 9 sensores. La diferente información obtenida por estos sensores se pueden utilizar para controlar en tiempo real el estado de un paciente o de obtener datos confidenciales con el fin de poste-riormente analizada para el diagnóstico médico (McCarthy, 1956) plantearon realizar el Aprendizaje progresivo basado en proyectos en el ámbito de la Ingeniería Biomédica: diseño, construcción y programa-ción de un ECG basado en un microcontrolador de (González M. 2005).

\section{CONCLUSIONES.}

Se presenta el análisis de los resultados obtenidos, de la clasificacion arrojados por la NNET y la SVM contrastados con los los valores reales tomados de la base de datos PSMEM . También se analiza la pertinencia e idoneidad del método empleado y hasta qué punto aporta unos resultados válidos y generalizables para el fin que se pretende. No existe un modelo clasificador mejor que otro de manera general. Así, para cada problema nuevo es necesario determinar con cuál clasificador se pueden obtener mejores resultados, y es por esto que han surgido varias medidas para evaluar la clasificación y comparar los modelos empleados para un problema determinado. Las medidas más conocidas para evaluar la clasificación están basadas en la matriz de confusión representadas por VP y FP, que se obtiene cuando se prueba el clasificador en un conjunto de datos que no intervienen en el entrenamiento.

El desarrollo de herramientas informáticas para el soporte de las decisiones clínicas tiene especial relevancia en los sistemas de salud del mundo que enfrentan retos de atención a sus poblaciones, en especial en el ejercicio clínico individual del día a día, donde los médicos enfrentan barreras de tiempo, conocimiento y costos, por esta razón el desarrollo de aplicaciones que disminuyan costos a los sistemas de salud tiene especial importancia en cualquier país del mundo. Este trabajo es una apuesta interesante por desarrollar una aproximación paraclínica al diagnóstico de la de individuos sanos y enfermos con sobrepeso u obesidad, que hoy por hoy sigue siendo un reto clínico. La predicción en medicina es un deseo generalizado de los médicos clínicos y afinar las herramientas que ayudan al diagnóstico mediante métodos estadísticos tiene un valor que trasciende a la seguridad de los pacientes, y por este solo hecho todos los estudios y proyectos que se desarrollen deben direccionarse a esta loable labor.

Este trabajo representa en Colombia un interesante inicio de la aproximación paraclínica a través de las tecnologías que sin duda a medida que se vaya refinando en datos estadísticos y eventual reducción en el número de variables, pueda tanto ayudar a mejorar la certeza diagnostica en función de la sensibilidad y como a aliviar los costos de exámenes innecesarios al sistema de salud. La 
ANN com FFBP sobre una tarjeta de desarrollo para estimación del fenotipo metabólico, el cual va a cumplir labor de apoyo paraclinico al diagnostico de los especialistas

\section{Referencias}

Álvarez L, R. y Arango M, A. (2013). "Implementación de la generación fotovoltaica Como respaldo ante fallos en la red de distribución". Revista Colombiana de Tecnologías de Avanzada, Vol 2, $\mathrm{N}^{\circ} 22$, p 14-19.

Álvarez L, R., García A, M. y Terregroza E, A. (2014). "Convertidores fotovoltaicos en módulos integrados basados en inversores de fuente - semi - cuasi - z: un nuevo esquema de control por modos deslizantes. Revista Colombiana de Tecnologías de Avanzada, Vol 1, $\mathrm{N}^{\circ}$ 23, p 61-66.

Beleño S, K., Pardo G, A. y Torres C, I. (2014). "control aplicado a una estructura mecánica Para el posicionamiento automático de módulos fotovoltaicos". Revista Colombiana de Tecnologías de Avanzada, Vol 2, $\mathrm{N}^{\circ} 22$, p 138-144.

Carignano F 2011. Tecnología inalámbrica Near Field Communication y sus aplicaciones en sistemas embebidos Especialidad en Sistemas Embebidos Instituto Universitario Aeronáutico Av. Fuerza Aérea 6500 - (5010) Universidad Nacional de Córdoba.

Colmenares, G. (2009). Inteligencia Artificial. En G. Colmenares, Maquinas de Vectores de Soporte.

Cybenko G. (1989). Appoximacion by Superpositions of Sigmoidal Function. Mathematics of control, Signals, ahd Systems 303-3014 Sprnger_Variag New York Inc.

Flores J, Barrera R., (2002). Entrenamiento de Máquinas de Soporte Vectorial. Universidad Autónoma del Carmen, Campeche, México.

González M. (2005). Aprendizaje Estadístico, Redes Neuronales y Support Vector Machines: Un enfoque global. Universidad Veracruzana, Xalapa, México.
Gunn S. (1997). Support Vector Machines for Classification and Regression. Universidad de Southampton.

Haykin S Neural Networks and Learning Machines Hamilton, Ontario, Canada ISBN-13: 978-013-147139-9 ISBN-10: 0-13-147139-2. Neural networks. II.Title. QA76.87.H39 2008 006.3--dc22 (1999).

Hilera J , Martínez V, "Redes Neuronales Artificiales", Alfa omega-Rama, 2000.

Leal G, F. y Hernandez C, M. (2013). "estudio del potencial eólico y solar de Cúcuta, Norte de Santander". Revista Colombiana de Tecnologías de Avanzada, Vol $2, \mathrm{~N}^{\circ} 22$, p 27-33.

Mandal, U., Banerjee, I., (2015) Cancer classification using neural network, International Journal of Emerging Engineering Research and Technology, 3(7): 172-178.

Mathworks (2008) INC gettingStarted Guide. The MathWorks, Inc.USA.

McCarthy, J (1956) Computer scientist known as the father of AI. John McCarthy, computer scientist: Died Stanford, California 24 October 2011.

Moreno, G. M. (2012). Definición y clasificación de la obesidad. Revista Médica Clínica Las Condes, 23(2), 124-128.

Olabe, X. B. (1998). Redes neuronales artificiales y sus aplicaciones. Publicaciones de la Escuela de Ingenieros 101pp.

Ramírez C. Universidad Nacional Experimental del Táchira. [Tesis Doctoral] Universidad de Surrey, Inglaterra. 1997.

Raponi M Bonnin R (2011) Investigador del Centro de Investigaciones en Láseres y Aplicaciones (CEILAP), perteneciente al Instituto de Investigaciones Científicas y Técnicas para la Defensa (CITEDEF).

Vides H, C., Pardo G, A. y Torres C, I. (2013). "Módulo robótico de seguimiento solar Usand o mppt supervisado y controlado vía Ethernet".Revista Colombiana de Tecnologías de Avanzada, Vol 1, N²3, p 112-119. 